\title{
Advances in the characterization, modelling and simulation of materials subjected to dynamic loading
}

\author{
Eric Buzaud $^{1, a}$, Antonio Cosculluela ${ }^{1}$, Hervé Couque ${ }^{2}$, and Ezio Cadoni ${ }^{3}$ \\ ${ }^{1}$ CEA CESTA, 15 avenue des Sablières, 33114 Le Barp Cedex, France \\ ${ }^{2}$ Nexter Munitions, 7 route de Guerry, 18023 Bourges, France \\ ${ }^{3}$ DynaMat Laboratory, University of Applied Sciences of Southern Switzerland, \\ 6952 Canobbio, Switzerland
}

Received 26 June 2018

Published online 10 september 2018

Materials constitute the foundations on which industries, economies and civilisations have always been built. For this reason, and this is what distinguishes materials from "matter", materials are by nature functional, designed by human beings to fulfil dedicated functions, and so to have a corresponding level of performance. Structural materials constitute a large class of materials designed primarily for their mechanical performance, but they also need to meet secondary performance criteria, such as weight, cost, recycling potential, durability, reliability, absence of toxicity, etc. Altogether, these performances can be reached through different manufacture processes which in turn affect distinctively the microstructural organisation and other physical properties of the resulting product.

Increasingly, the development of materials by design includes considerations relative to their mechanical behaviour under dynamic loadings, which can be considered as a performance factor itself, or conversely, be solely observed as a consequence of other performance criteria, beneficial or detrimental to their response in those particular conditions. The engineer must determine the capability of the structure to sustain this class of loadings, and in case irreversible deformations would be predicted, it is often necessary to determine the level of transformation, from slight local damage or plasticity to complete collapse. Civil engineering, defence, aeronautical and transportation industries constitute the principal frameworks of this kind of safety and protection analysis.

All materials respond to a dynamic loading through the propagation of stress waves, and for most of them, this response is affected by the rate of the loading; strength enhancement and embrittlement being one of the most commonly observed phenomena. This sensitivity to the rate of loading is itself affected by other factors. Some of them are related to additional characteristics of the loading such as ambient temperature and hydrostatic pressure, triaxiality and direction of loading (compression, traction, shear). Some others are related to long term alteration of the initial properties: wear, slow chemical reactions, radiation damage, evaporation of some phases, etc.

Understanding and quantifying those effects and their combinations have been a research field of growing importance since the 1970s, driven by the need for predictive

a e-mail: eric.buzaud@cea.fr 
models linking material microstructural properties to in-service structural response. Considerable progress has been achieved in recent years, due on the one hand to new experimental techniques, improved precision high rate diagnostics, conceptual multiscale modelling (made possible thanks to ever increasing computational power), and on the other hand to the growing development of innovative materials by scientific design.

In the contributions collected in this issue of EPJ-ST, essentially three large classes of structural materials are addressed: concrete, polymers and metals. The recent advances mentioned above are illustrated by 15 papers selected from a total of 200 contributions through a rigorous peer review process. All 15 papers were presented at the 12th International Conference of the DYMAT Association, the European association for the promotion of research into the dynamic behaviour of materials and its applications, held in Arcachon, France, in September 2018 and organized under the auspices of the Commissariat à l'Energie Atomique et aux Energies Alternatives. 